\title{
Short-Term Effects of a Multimodal 3-Week Inpatient Pulmonary Rehabilitation Programme for Patients with Sarcoidosis: The ProKaSaRe Study
}

\author{
Heidrun Lingner ${ }^{a}$ b Heike Buhr-Schinner ${ }^{c}$ Stefan Hummel ${ }^{d}$ \\ Jochen van der Meyden ${ }^{\mathrm{e}}$ Anika Grosshennig ${ }^{f}$ Daniel Nowik ${ }^{g} \quad$ Konrad Schultz $^{\text {h }}$

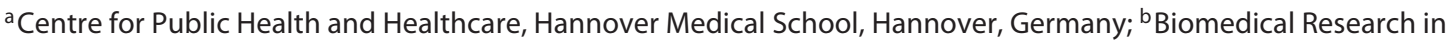 \\ Endstage and Obstructive Lung Disease Hannover (BREATH), German Center for Lung Research (DZL), Hannover,

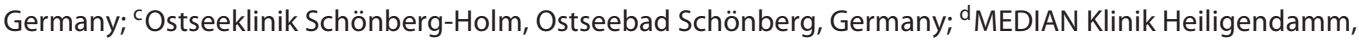

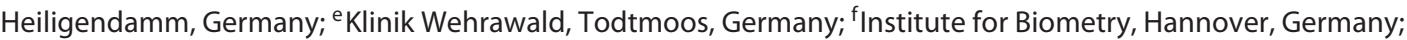 \\ gInstitute for Epidemiology, Social Medicine and Health Systems Research, Hannover Medical School, Hannover,

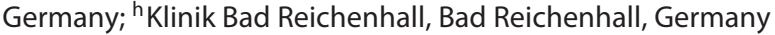

\section{Keywords}

Sarcoidosis · Pulmonary rehabilitation · Quality of life · Fatigue

\begin{abstract}
Background: Real-world data on the effects of a multicomponent pulmonary rehabilitation (PR) for patients with sarcoidosis are scarce. Objective: To describe characteristics of patients with sarcoidosis referred for a 3-week inpatient PR, to assess the effects of PR on their quality of life (QoL) and clinical outcomes, and to investigate whether there are specific subgroups who particularly benefit from PR. Methods: Using a prospective multicentre study design, data regarding 6-min walking distance (6MWD), QoL (Saint George's Respiratory Questionnaire, SGRQ), and the secondary outcomes of dyspnoea and psychological burden (fatigue, anxiety, and depression) were collected. Results: We included 296 patients in the study (average age $49.1 \pm 9.7$ years, $47 \%$ female, average vital capacity $3.5 \pm 1.0 \mathrm{~L}[87.0 \pm 20.6$ predicted]). The $6 \mathrm{MWD}$ improved by the end of the rehabilitation by $39.8 \mathrm{~m}$ on average $(p<0.0001$; standardised response
\end{abstract}

(C) 2018 S. Karger AG, Basel

\section{E-Mail karger@karger.com www.karger.com/res \\ KARGER}

mean, SRM $=0.61)$, SGRQ showed significant improvements in all 3 domains, and the total score $(p<0.001)$ improved by $5.69-8.28$ points (SRM $0.46-0.62$ ). For the secondary outcomes, significant improvement $(p<0.001)$ was seen for all measured parameters, e.g., dyspnoea (modified Medical Research Council Scale, mMRC), fatigue (Fatigue Assessment Scale [FAS]; SRM = -0.71), anxiety and depression (Hospital Anxiety and Depression Scale [HADS]; SRM -0.58/-0.38), and generic QoL (measured by the SF-36 scales of physical and mental health; SRM 0.31/0.55). Conclusions: Our results provide the first documented evidence that $P R$ is a promising complementary therapy option for sarcoidosis patients who remain subjectively symptomatic despite optimised outpatient medical treatment.

(c) 2018 S. Karger AG, Basel

\section{Introduction}

Sarcoidosis is a systemic granulomatous disease of unclear aetiology. It presents with heterogeneous clinical symptoms which determine its impact. While $90 \%$ of pa- 
tients with acute sarcoidosis recover, chronically affected patients may remain asymptomatic (up to $50 \%$ ), but 10 $20 \%$ [1] suffer from permanent sequelae (pulmonary or extrapulmonary), serious physical disability, highly reduced quality of life (QoL), and intense fatigue [2]. Approximately $90 \%$ of patients have pulmonary involvement.

Pulmonary rehabilitation (PR) is an appropriate treatment for many chronic respiratory diseases [3-8]. However, the available data regarding PR for sarcoidosis are very sparse compared to the evidence for the benefits of PR for COPD patients. Although PR is indicated for sarcoidosis in the international (PR) guidelines, no specific studies are cited as evidence $[1,3]$. Sarcoidosis patients have been included in some randomised controlled trials (RCTs) focusing on the effectiveness of physical training or multimodal PR for interstitial lung diseases $[9,10]$ and a series of observational studies $[11,12]$. These studies, however, used mixed patient groups and only presented partial results for the sarcoidosis subgroup. This paucity of data is surprising, particularly as the topic of muscle weakness and training has often been discussed in the literature [13-18].

Current papers recommend PR for sarcoidosis patients [19], particularly those with muscle weakness. The goal of this study is to assess and discuss the short-term changes in pulmonary and QoL parameters in patients with sarcoidosis undergoing a multimodal and interdisciplinary PR.

\section{Study Design and Methods}

The data used in this study originate from the ProKaSaRe study (Prospective Katamnesis Study regarding the Course of Sarcoidosis in Pulmonary Rehabilitation), a prospective, multicentric, observational study. This project was supported by the rehabilitation section of the German Respiratory Society (DPG). Ethics committee approval from the Hannover Medical School was received prior to study initiation (No. 877). The study was registered with the German Clinical Trials Register (ID: DRKS00000560).

\section{Sampling: Patient Population}

All consecutive patients referred to one of the six participating German PR clinics between January 2011 and January 2012 for inpatient rehabilitation due to sarcoidosis were invited to participate in the ProKaSaRe. They were included in the study if they provided informed consent and had a confirmed diagnosis of sarcoidosis by a respiratory specialist. Patients incapable of completing the questionnaire due to cognitive disability or lack of German language skills were excluded. The obligatory and optional PR components performed at all study centres for the 3-week inpatient PR are described in Table 1. Primary and secondary outcomes were assessed at the start (T0) and end (T1) of PR as follows:

\section{Primary Outcomes}

1. Disease-specific QoL was assessed with the St. George's Respiratory Questionnaire (SGRQ). This questionnaire was initially developed to quantify QoL in patients with respiratory disorders [20] and has also been used for sarcoidosis patients [21, 22]. Values for the total score and the three subscores (symptoms, activity, and impact) range from 0 (no impairment) to 100 (maximum impairment). A minimal clinically important difference (MCID) of 4 points in the total score has been shown for COPD and asthma [23], but no MCID value exists for sarcoidosis.

2. The standardised Six-Minute Walk Test (6MWT) is used for respiratory diseases [24] to evaluate physical fitness and is recommended for the assessment of sarcoidosis patients $[1,16$, 25]. A test-specific MCID for patients with sarcoidosis has not yet been defined.

\section{Secondary Outcome Parameters}

Generic QoL was evaluated using the German version of the SF-36 (36-item Short Form Health Survey Questionnaire) [26], which examines self-assessed psychological, physical, and social aspects of QoL. Out of 9 questionnaire sections, 4 address physical health, 4 address subjective psychological health, and 1 covers the change in health over time. Scores from 0 (worst) to 100 (best) points can be obtained.

Fatigue, the most common sarcoidosis symptom, was measured using the Fatigue Assessment Scale (FAS) [27]. This scale contains 10 items with a possible total score of up to 50 points. While scores of 22-34 indicate mild to moderate fatigue, higher values indicate extreme fatigue. Clinical symptoms such as fever, sputum, cough, breathlessness, joint pain, eye disorders, and lymph node swelling were systematically documented at T0 and T1 using a standardised questionnaire. The severity of the symptoms was quantified by using a Likert scale from 1 to 5 (very low to very high).

The psychological strain of patients was measured using the German version of the Hospital Anxiety and Depression Scale (HADS-D [28]). The questionnaire comprises 7 questions about anxiety and depression, each with specifically formulated possible answers on a 4-point scale (scoring 0-3 points). A total score of up to 7 points is considered normal, $8-10$ points signify the borderline, and 11 points and above indicate psychological disorders.

Lung function was assessed with spirometry and body plethysmography (Masterlab; Jaeger, Würzburg, Germany) according the recommendations of the German guidelines $[29,30]$. The following parameters were examined: vital capacity (VC [litres, \% predicted]), residual volume (litres, $\%$ predicted), total lung capacity (litres, \% predicted), forced expiratory volume in $1 \mathrm{~s}\left(\mathrm{FEV}_{1}\right.$ [litres, \% predicted]), total specific resistance $(\mathrm{kPa} \bullet \mathrm{s}, \%$ predicted), Tiffeneau index (each before and after administration of bronchodilators), and respiratory muscle function (P 0.1 und PI $\max [\mathrm{kPa}])$.

Further parameters examined included gas exchange, assessed by the diffusing capacity of the lung for carbon monoxide $\left(\mathrm{D}_{\mathrm{LCO}}\right)$. Additionally, $\mathrm{PaO}_{2}$ and $\mathrm{PaCO}_{2}$ were determined at rest and during exercise at $50 \mathrm{~W}$ in order to detect impaired lung diffusion. Severity of dyspnoea was documented using the modified Medical Research Council Scale (mMRC) [31], scoring from 0 (breathless only during strenuous exercise) to 4 (not possible to leave home 
Table 1. Obligatory and optional components of pulmonary rehabilitation (PR)

Obligatory PR components (completed by all patients):

Confirmation of the diagnosis by a lung specialist at admission to PR; the previously prescribed long-term sarcoidosis medication was usually not changed

Endurance training: 3-5 units/week (each $30-60 \mathrm{~min}$ ) at approx. $60-80 \%$ of the maximum capacity

(walking, Nordic walking, or use of a bicycle ergometer)

Strength training: 3 sessions/week (each 30-60 min)

Respiratory physiotherapy: 3 group sessions/week and at least 2 individual respiratory physiotherapy sessions during the PR

Patient education for sarcoidosis and other optional education modules (e.g., patient education concerning long-term oxygen therapy)

Optional PR components (prescribed as required by the clinic doctors):

Relaxation therapy: autogenic training or progressive muscle relaxation

Inspiration muscle training: $5 \times 30 \mathrm{~min} /$ week, starting at 30\% PI max, supervised at least once per week

Social counselling

Nutrition counselling

Psychological support (individual and group therapy)

Structured smoking cessation programme

Ergotherapy and advice on the use of aids and appliances

due to breathlessness, or breathlessness when dressing). Levels of angiotensin-converting enzyme (ACE), produced in sarcoidosis by epithelioid cells of granulomas, were used to determine and follow granuloma load as approximately $40-80 \%$ of sarcoidosis patients show increased ACE levels [32].

\section{Analysis}

Independent double data entry for all anonymised questionnaires and documents was performed at the Hannover Medical School by individuals not involved in patient care. As ProKaSaRe was an exploratory study, parameters and clinical characteristics were evaluated descriptively and considered to be important at $p \leq 0.05$; no adjustments for multiplicity or imputation of missing data were performed. Changes from baseline for continuous data were analysed using two-sided paired $t$ tests and the mean differences (T0-T1) with 95\% confidence intervals (95\% CI) are provided. We used regression analysis to estimate the influences of possible confounders such as age, gender, obesity, change of medication, or comorbidity on the primary outcomes. To rule out possible effects of obesity and sarcoidosis severity (as assessed by X-ray) on fatigue and dyspnoea, we calculated $\chi^{2}$ tests for associations between $\mathrm{X}$-ray type and obesity, fatigue, and dyspnoea and compared paired $t$ tests for changes in fatigue between obese and non-obese patients. We also intended to perform subgroup analysis using linear regression to contrast primary outcomes and the core symptom of sarcoidosis, fatigue, between the most severely physically impaired patients and the complementing sample.

All statistical analyses were conducted using SAS 9.3 software (SAS Institute Inc., Cary, NC, USA). Methods regarding sample size calculation and statistical procedures have been described previously [33].

\section{Results}

\section{Description of the ProKaSaRe Sample}

Patients were recruited for ProKaSaRe between January 2011 and January 2012. Written informed consent was provided by 296 patients; $47.0 \%$ were female, with an average age of $49.1 \pm 9.7$ years. Average BMI was $28.7 \pm$ 5.9 and thus most patients were either overweight $(32.0 \%$, BMI 25-30) or obese (39.6\%, BMI $\geq 30)$. According to available chest $\mathrm{X}$-rays, $85.1 \%$ of patients were classified as $\mathrm{X}$-ray type 0-IV [34]. With respect to the involved areas of the lung, the distribution of X-ray types 0 -IV was as follows: $1.5,18.3,58.0,13.7$, and $4.6 \%$, respectively. Further parameters are shown in Table 2.

In addition to the lung or the intrathoracic lymph node involvement observed in all study participants, the following organs were also affected: skin (13.9\%), bones/ joints $(13.9 \%)$, eyes $(6.8 \%)$, heart $(4.4 \%)$, liver $(4.1 \%)$, muscles (2.7\%), CNS (3.7\%), kidneys (1.4\%), and peripheral nerves $(1.0 \%)$. The three most common comorbidities in the study sample were obesity (39.6\%), arterial hypertension (37.8\%), und degenerative spine disorders (27.0\%). Further comorbidities included depression (13.9\%), diabetes (9.5\%), osteoarthrosis (7.4\%), sleeprelated breathing disorders $(6.8 \%)$, and cardiac arrhyth$\operatorname{mia}(6.4 \%)$.

Nearly half of the ProKaSaRe patients $(48.8 \%)$ were currently receiving systemic steroids, $55.8 \%$ had been previously treated with them, and $9.3 \%$ of the patients 
Table 2. Baseline characteristics

\begin{tabular}{|c|c|}
\hline Age (range), years & $49.1 \pm 9.7(23-79)$ \\
\hline Female, $\%(n / N)$ & $47.0(139 / 296)$ \\
\hline BMI & $28.7 \pm 5.9(12.4-48.4)$ \\
\hline IVC, L & $3.5 \pm 1.0(87.0 \pm 20.6)$ \\
\hline $\mathrm{FEV}_{1}, \mathrm{~L}$ & $2.8 \pm 0.9(85.1 \pm 21.6)$ \\
\hline $\mathrm{FEV}_{1} / \mathrm{VC}, \%$ & $78.5 \pm 12.7$ \\
\hline SRtot, $\mathrm{kP} \bullet \mathrm{s}$ & $1.0 \pm 0.9(100.1 \pm 70.0)$ \\
\hline $\mathrm{PI} \max , \mathrm{kPa}$ & $6.8 \pm 3.3(74.6 \pm 46.7)$ \\
\hline $\mathrm{P} 0.1, \mathrm{kPa}$ & $0.3 \pm 0.2(105.2 \pm 62.3)$ \\
\hline $\mathrm{PaO}_{2}$ at rest, $\mathrm{mm} \mathrm{Hg}$ & $78.9 \pm 11.0$ \\
\hline $\mathrm{PaCO}_{2}$ at rest, $\mathrm{mm} \mathrm{Hg}$ & $36.0 \pm 5.1$ \\
\hline $\mathrm{PaO}_{2} 50 \mathrm{~W}, \mathrm{~mm} \mathrm{Hg}$ & $78.1 \pm 12.4$ \\
\hline $\mathrm{PaCO}_{2} 50 \mathrm{~W}, \mathrm{~mm} \mathrm{Hg}$ & $36.1 \pm 5.0$ \\
\hline $\mathrm{D}_{\mathrm{LCO}} \mathrm{SB}, \mathrm{mmol} / \mathrm{min} / \mathrm{kPa}$ & $7.6 \pm 2.7(80.2 \pm 24.1)$ \\
\hline $\mathrm{D}_{\mathrm{LCO}} / \mathrm{VA}, \mathrm{mmol} / \mathrm{min} / \mathrm{kPa} / \mathrm{L}$ & $1.6 \pm 0.7(94.9 \pm 23.2)$ \\
\hline $\mathrm{ACE}, \mathrm{U} / \mathrm{L}$ & $46.2 \pm 45.0$ \\
\hline
\end{tabular}

Values are mean \pm SD (\% predicted) unless otherwise indicated. IVC, inspiratory vital capacity; SRtot, total specific resistance; SB, single breath; VA, alveolar volume.

were receiving other immunosuppressive treatments. Emergency inpatient or outpatient treatment for sarcoidosis during the previous year was reported by 40.9 and $27.4 \%$ of patients, respectively.

\section{Primary Outcomes}

After PR, the average increase in 6-min walking distance (6MWD) was $39.8 \mathrm{~m}$ (standardised response mean, $\mathrm{SRM}=0.61$; see Table 3), indicating a medium effect size. Heart rate, oxygen saturation at rest and during exercise, and perceived breathlessness at the end of the 6MWT (Borg Scale) did not change significantly.

The average scores and changes in the SGRQ at T0 und T1 (see Table 4) were calculated only for patients with complete paired data. Significant improvements were seen in all domains, most clearly for "symptoms" and "activity" (8.3 and 7.8 points, respectively), corresponding to a medium effect in both domains.

Regression analysis showed a positive association of anxiety, heart rhythm disorders, and male gender with the changes in $6 \mathrm{MWD}$, while initial walking distance was the strongest predictor for gain in distance from T0 to T1.

Regarding QoL, a model showed a negative association of depression, spinal disorders, and hypertension with the total SGRQ score. Total SGRQ score at T0 was the strongest predictor for changes in QoL. Other variables such as comorbidities including obesity, age, steroid treatment, and optimisation of medication did not have any statistically significant influence on 6MWD or QoL in our sample.

With the aim of increasing the specificity of the results, we tried to assess the changes in the most severely affected group of patients, but due to the low total number $(n=14)$ with severe physical impairment (defined by the lowest $10 \%$ of $6 \mathrm{MWD}$, and/or the lowest FVC und FEV1, and/or in need of $\mathrm{O}_{2}$ ), no contrasting analysis could (or should) be performed. Differences, even if existing, would not show any significance and the results of such analysis would lead to inaccurate assumptions. We have therefore postponed further investigations of the subgroups until the planned upcoming waiting-list design RCT can be performed.

\section{Secondary Outcome Parameters}

Table 5 shows changes in lung function parameters, respiratory muscle strength, blood gas analyses at rest and during exercise, diffusion capacity, and ACE. A significant reduction in mean ACE level from $46.2 \mathrm{U} / \mathrm{l}$ at T0 to $44.4 \mathrm{U} / \mathrm{l}$ at $\mathrm{T} 1$ was observed. Small but significant changes were also seen in lung function according to $\mathrm{VC}$ and $\mathrm{FEV}_{1}$ measurements. However, SRM $(\sim 0.2)$ indicated a small effect size for both outcomes, thus these findings were interpreted as clinically irrelevant. The average Tiffeneau index had normal values before and after PR and showed no significant change. The average maximum static inspiratory pressure (PI max) was reduced at T0, but was normal by the end of PR $(S R M=0.4)$. Mean blood gas values at rest and during exercise at $50 \mathrm{~W}$ and $\mathrm{D}_{\text {LCO }}$ values were normal at T0 (Table 1) and did not change significantly by $\mathrm{T} 1$.

Values of (subjectively assessed) fatigue (FAS), anxiety, depression (HADS), (generic) QoL (SF-36), and dyspnoea (MRC) at baseline and at the end of PR are shown in Table 4. The mean FAS score improved significantly between $\mathrm{T} 0$ and $\mathrm{T} 1$ by 4.09 points, indicating a medium effect size (SRM = 0.71). Since the MCID [35] for FAS is 4 points, the mean improvement was clinically relevant and was achieved by $39.2 \%$ of patients. Clinically relevant fatigue (FAS $\geq 22$ ) was reported by $65.9 \%$ of patients at $\mathrm{T} 0$ but only by $44.3 \%$ at T1. Moreover, FAS score changes correlated with changes in SGRQ, 6MWD, and MRC dyspnoea scores. Both obese and non-obese patients showed a significant reduction of fatigue. A significant decrease of fatigue was also assessed in patients classified as type 0 -II via X-ray. More severely affected patients (type III and IV) did not report reduced fatigue $(t=0.956, \mathrm{df}=35$, $p \leq 0.346)$. Psychological and physical health scores, however, improved at the end of PR, with low or medium ef- 
Table 3. Six-Minute Walk Test (6MWT) at T0 and T1

\begin{tabular}{|c|c|c|c|c|c|c|c|}
\hline \multirow[t]{2}{*}{$6 \mathrm{MWT}$} & \multicolumn{2}{|l|}{ T0 } & \multicolumn{5}{|c|}{ Change (T1-T0) } \\
\hline & mean & SD & $\Delta$ mean & $\begin{array}{l}95 \% \text { CI } \\
\text { lower } \\
\text { limit }\end{array}$ & $\begin{array}{l}95 \% \text { CI } \\
\text { upper } \\
\text { limit }\end{array}$ & $p$ value & $\begin{array}{l}\text { SRM } \\
(\mathrm{T} 0-\mathrm{T} 1)\end{array}$ \\
\hline $6 \mathrm{MWD}, \mathrm{m}$ & 505.2 & 112.2 & +39.8 & +31.9 & +47.7 & $<0.0001$ & +0.61 \\
\hline Heart rate at rest & 80.2 & 12.5 & -1.23 & -2.59 & +0.13 & 0.0771 & -0.11 \\
\hline Heart rate during exercise & 112.0 & 20.0 & +0.23 & -1.94 & +2.40 & 0.8364 & +0.01 \\
\hline $\mathrm{O}_{2}$ saturation at rest & 96.5 & 2.6 & +0.14 & -0.17 & +0.45 & 0.3661 & +0.05 \\
\hline $\mathrm{O}_{2}$ saturation during exercise & 94.1 & 5.3 & +0.26 & -0.22 & +0.74 & 0.2903 & +0.06 \\
\hline Borg score & 3.3 & 1.9 & -0.14 & -0.31 & +0.04 & 0.1237 & -0.099 \\
\hline
\end{tabular}

Measurements are shown for the 262 patients for whom data were available at T0 und T1. 6MWD, 6-min walking distance; SRM, standardised response mean. ${ }^{a}$ At the end of the $6 \mathrm{MWT}$.

Table 4. Changes in (generic) quality of life after pulmonary rehabilitation in FAS, HADS, and SF-36

\begin{tabular}{|c|c|c|c|c|c|c|c|}
\hline & \multicolumn{2}{|l|}{ T0 } & \multicolumn{5}{|c|}{ Change (T1-T0) } \\
\hline & mean & $\mathrm{SD}$ & $\Delta$ mean & $\begin{array}{l}95 \% \text { CI } \\
\text { lower } \\
\text { limit }\end{array}$ & $\begin{array}{l}95 \% \text { CI } \\
\text { upper } \\
\text { limit }\end{array}$ & $\mathrm{p}$ value & $\begin{array}{l}\text { SRM } \\
\text { (T0-T1) }\end{array}$ \\
\hline FAS (10-50) & 26.6 & 7.7 & -4.09 & -4.82 & -3.36 & $<0.0001$ & -0.71 \\
\hline HADS: anxiety & 7 & 3.4 & -1.56 & -1.88 & -1.24 & $<0.0001$ & -0.58 \\
\hline HADS: depression & 7.7 & 2.9 & -0.94 & -1.24 & -0.65 & $<0.0001$ & -0.38 \\
\hline SF-36: physical health & 39.3 & 10.6 & 2.0 & 1.18 & 2.87 & $<0.0001$ & 0.31 \\
\hline SF-36: psychological health & 42.5 & 11.7 & 5.3 & 4.03 & 6.55 & $<0.0001$ & 0.55 \\
\hline \multicolumn{8}{|l|}{ SGRQ } \\
\hline Symptoms & 43.4 & 24.4 & -8.28 & -10.61 & -5.95 & $<0.0001$ & -0.46 \\
\hline Activity & 49.4 & 23 & -7.76 & -9.53 & -6.00 & $<0.0001$ & -0.56 \\
\hline Impact & 29.9 & 19.9 & -5.69 & -7.23 & -4.15 & $<0.0001$ & -0.48 \\
\hline Total Score & 38.3 & 19.8 & -6.66 & -8.09 & -5.22 & $<0.0001$ & -0.62 \\
\hline
\end{tabular}

Average \pm SD for the 3 SGRQ sections and the total scores at T0 and T1, as well as the average changes from T0 to T1 with 95\% CI, $p$ values, and effect sizes. FAS, Fatigue Assessment Scale; HADS, Hospital Anxiety and Depression Scale; SF-36, 36-item Short Form Health Survey Questionnaire; SGRQ, Saint George's Respiratory Questionnaire; SRM, standardised response mean.

fect sizes (see Fig. 1), but remained below the scores of healthy people.

Table 6 shows clinical symptoms described by at least a third of patients at T0. The most commonly reported symptom was exercise intolerance (92.9\%). Of these patients, $46.9 \%$ rated this impediment as "strong" or "very strong." At the end of rehabilitation only $78 \%$ of patients still reported exercise intolerance and only $21.3 \%$ of them indicated that they still felt strongly or very strongly limited in their ability to exercise.

Reports of dyspnoea decreased slightly from $78.5 \%$ at T0 to $71.2 \%$ at T1, but only $15.5 \%$ of dyspnoea was grad- ed as severe or extremely severe at T1 (T0: $33.5 \%)$. The distribution of MRC dyspnoea scores improved significantly, as shown in Figure $2\left(\chi^{2} 213.35, p \leq 0.001\right)$. Patients showed significant and positive improvements in dyspnoea, regardless of X-ray classification or obesity.

The mean improvement in HADS anxiety scores after PR was 1.6 points, indicating a medium effect size, and at T1, only $19.9 \%$ and $7.8 \%$ of patients had values of $8-10$ or $\geq 11$, respectively. The average HADS depression score was 7.7 points at $\mathrm{T} 0$, indicating an elevated average degree of depressive symptoms which improved by 0.94 points by $\mathrm{T} 1$. Clinically relevant depression at T0 was reported 
Table 5. Changes in the parameters for spirometry, body plethysmography, tests of respiratory muscle strength and gas exchange at rest and during exercise at $50 \mathrm{~W}(\mathrm{~T} 0 \rightarrow \mathrm{T} 1)$, lung diffusion, and ACE

\begin{tabular}{|c|c|c|c|c|c|}
\hline \multirow[t]{2}{*}{ Variable } & \multicolumn{4}{|c|}{ Change (T1-T0) } & \multirow{2}{*}{$\begin{array}{l}\text { SRM } \\
\text { (T0-T1) }\end{array}$} \\
\hline & $\Delta$ mean & $\begin{array}{l}95 \% \text { CI } \\
\text { lower limit }\end{array}$ & $\begin{array}{l}95 \% \text { CI } \\
\text { upper limit }\end{array}$ & $p$ value & \\
\hline IVC, L & +0.08 & +0.04 & +0.12 & $<0.0001$ & +0.24 \\
\hline IVC, $\%$ predicted & +2.58 & +1.39 & +3.76 & $<0.0001$ & +0.26 \\
\hline $\mathrm{FEV}_{1}, \mathrm{~L}$ & +0.08 & +0.04 & +0.11 & 0.0002 & +0.22 \\
\hline $\mathrm{FEV}_{1}, \%$ predicted & +1.79 & +0.77 & +2.82 & 0.0007 & +0.20 \\
\hline $\mathrm{FEV}_{1} / \mathrm{VC}, \%$ & +0.26 & -0.55 & \pm 1.07 & 0.5319 & +0.04 \\
\hline SRtot, $\mathrm{kP} \bullet \mathrm{s}$ & -0.04 & -0.13 & +0.05 & 0.3894 & -0.05 \\
\hline SRtot, \% predicted & +1.16 & -3.50 & +5.82 & 0.6248 & +0.03 \\
\hline PI max, $\mathrm{kPa}$ & +0.80 & +0.56 & +1.03 & $<0.0001$ & +0.40 \\
\hline PI max, \% predicted & +12.41 & +8.12 & +16.70 & $<0.0001$ & +0.40 \\
\hline $\mathrm{P} 0.1, \mathrm{kPa}$ & +0.03 & 0.00 & +0.05 & 0.0165 & +0.14 \\
\hline P $0.1, \%$ predicted & +6.88 & +0.33 & +13.44 & 0.0396 & +0.14 \\
\hline P 0.1/PI max, \% & -0.39 & -1.10 & +0.33 & 0.2925 & -0.07 \\
\hline $\mathrm{PaO}_{2}, \mathrm{~mm} \mathrm{Hg}$ at rest & +0.68 & -0.34 & +1.71 & 0.1908 & +0.08 \\
\hline $\mathrm{PaCO}_{2}, \mathrm{~mm} \mathrm{Hg}$ at rest & +0.08 & -0.51 & +0.66 & 0.7979 & +0.02 \\
\hline $\mathrm{D}_{\mathrm{LCO}} \mathrm{SB}, \mathrm{mmol} / \mathrm{min} / \mathrm{kPa}$ & +0.13 & -0.13 & +0.38 & 0.3237 & +0.06 \\
\hline $\mathrm{D}_{\mathrm{LCO}} \mathrm{SB}, \%$ predicted & +0.49 & -1.63 & +2.60 & 0.6495 & +0.03 \\
\hline $\mathrm{D}_{\mathrm{LCO}} / \mathrm{VA}, \mathrm{mmol} / \mathrm{min} / \mathrm{kPa} / \mathrm{L}$ & +0.01 & -0.08 & +0.11 & 0.7689 & +0.02 \\
\hline $\mathrm{D}_{\mathrm{LCO}} / \mathrm{VA}, \%$ predicted & -0.47 & -2.64 & +1.70 & 0.6689 & -0.03 \\
\hline $\mathrm{ACE}, \mathrm{U} / \mathrm{L}$ & -2.27 & -3.70 & -0.84 & 0.002 & -0.197 \\
\hline
\end{tabular}

IVC, inspiratory vital capacity; SRtot, total specific resistance; SB, single breath; VA, alveolar volume; SRM, standardised response mean.

Table 6. Frequencies (\%) of clinical symptoms at T0 and T1 which were rated as high or very high on a 5-point Likert Scale

\begin{tabular}{lllll}
\hline $\begin{array}{l}\text { Main clinical } \\
\text { symptoms }\end{array}$ & T0 & $\begin{array}{l}\text { High and } \\
\text { very high } \\
\text { severity }\end{array}$ & T1 & $\begin{array}{l}\text { High and } \\
\text { very high } \\
\text { severity }\end{array}$ \\
\hline Exercise intolerance & 92.9 & 46.9 & 78.0 & 21.3 \\
Fatigue & 83.9 & 40.3 & 72.4 & 22.3 \\
Dyspnoea & 78.5 & 33.5 & 71.2 & 15.5 \\
Joint pain & 60.2 & 39.1 & 46.5 & 27.6 \\
Cough & 55.8 & 13.6 & 49.3 & 7.5 \\
Mouth dryness & 35.6 & 23.4 & 35.6 & 16.3 \\
Skin changes & 33.8 & 25.3 & 24.5 & 20.9 \\
Eye disorders & 34.7 & 16.9 & 27.6 & 22.4 \\
\hline
\end{tabular}

by $28.4 \%$ ( $8-10$; borderline) and $15.9 \%$ (>11; abnormal) of the patients. At T1, these values were reduced to 20.6 and $11.5 \%$, respectively.

Because of its great clinical importance, we contrasted the secondary outcome of fatigue (FAS) between the se- verely physically impaired patients and the complementing sample, even though the sample size was very low. When calculating a regression analysis for the patients belonging either to the sample with the poorest lung function or with the shortest walking distance, adjusted for fatigue at baseline, we found that the most impaired showed a statistically significantly greater improvement in fatigue after PR than the other patients (a difference of 2.7-2.8 on the FAS scale). This is, however, only a small effect and due to the low number of patients in this subgroup, it must be analysed in a larger sample in order to allow firm conclusions to be drawn and, if confirmed, to investigate its clinical significance.

\section{Discussion}

The ProKaSaRe study described and characterised the "average" patient allocated to PR because of sarcoidosis. This "average" patient was sent for PR by the referring doctors and additionally underwent the rigorous exami- 


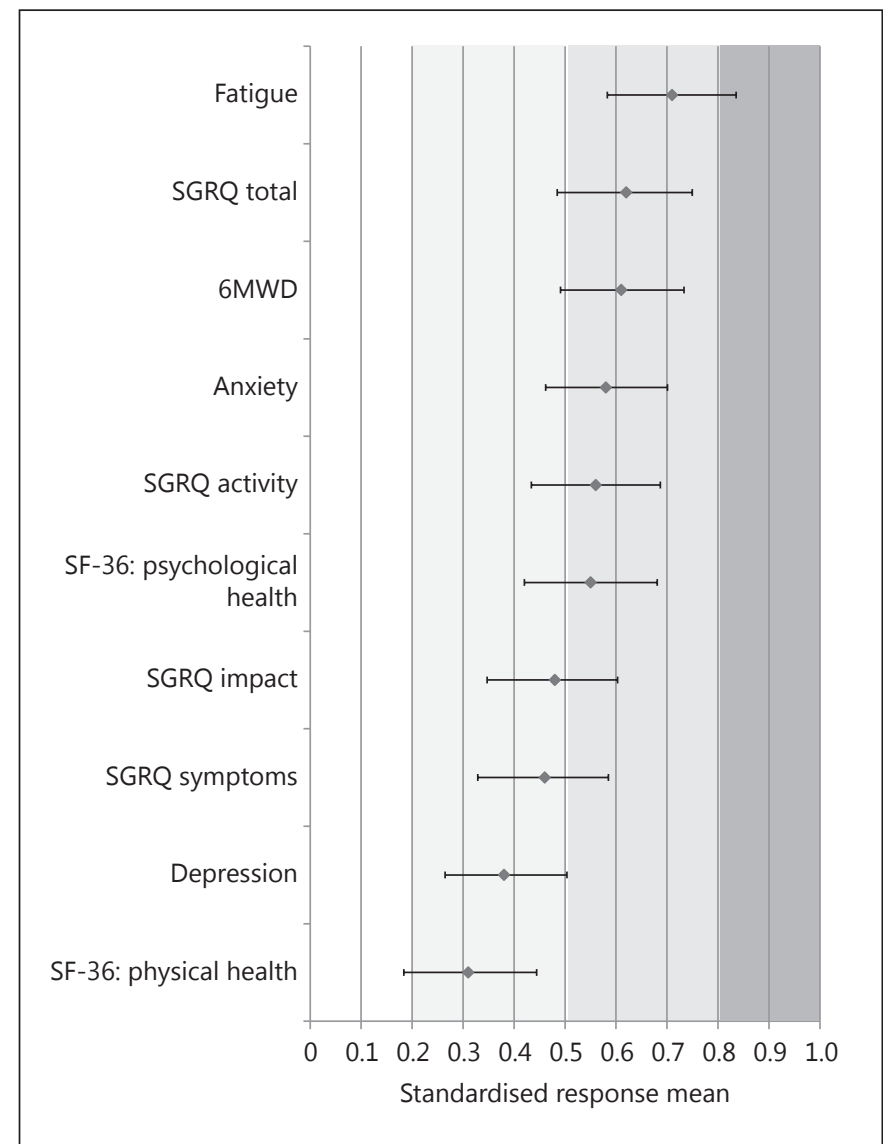

Fig. 1. Effect sizes (standardised response means) after rehabilitation for primary and secondary outcomes. SGRQ, Saint George's Respiratory Questionnaire; 6MWD, 6-min walking distance; SF-36, 36-item Short Form Health Survey Questionnaire.

nation required by the German Statutory Pension Insurance Scheme fund, resulting in a positive evaluation. In our sample, $48.8 \%$ of patients received continuous systemic medication with oral corticosteroids and $40.9 \%$ had been hospitalised or treated by emergency services during the previous months. Nevertheless, only a few had retired $(n=21)$ or were on sick leave $(n=75)$ upon their enrolment in our study (T0) and even fewer had severe sarcoidosis as characterised by X-ray stage or dyspnoea (20\%). Nevertheless, these patients do seem to need PR despite their "light sarcoidosis", pulmonary limitations apparently not being commonly the main reason for the referral for PR. We assume that the referrals are, instead, mainly due to frequent absences from work and the pronounced impairment the patients feel.

After a 3-week multidisciplinary inpatient rehabilitation, statistically significant and clinically relevant im-

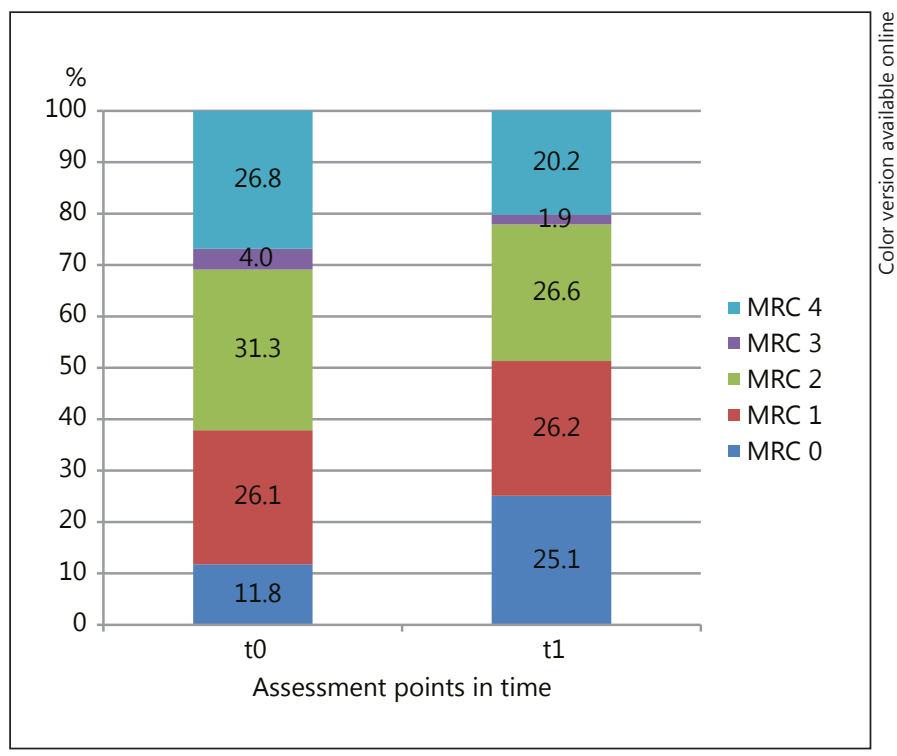

Fig. 2. Distribution of Medical Research Council Scale (MRC) scores before and after rehabilitation.

provements in functional exercise capacity (6MWD) and health-related QoL (HrQoL; SGRQ) were assessed. Fatigue, anxiety, depression, and dyspnoea (mMRC) also improved significantly, justifying the referrals retrospectively. These results are based on the largest number of participants in any rehabilitation study on this topic performed worldwide.

Physical fitness of participants at T0, as indicated by the mean 6MWD, was below the healthy average (at $505.2 \mathrm{~m}$ ) [36]. This corresponds to results from other studies of sarcoidosis patients [14, 15, 37-39]. The improvement in 6MWD of $39.8 \mathrm{~m}$ found in ProKaSaRe seems low at first but was perceived by patients as clinically relevant, according to individual feedback. Currently no MCID exists for 6MWD in sarcoidosis patients $[14,15]$. Two other publications reporting improvements in 6MWD in a comparable study population assessed a longer outpatient training programme. A recent Cochrane analysis of PR in interstitial lung disease [10] reported a mean difference pre-post PR in distance walked of $44.34 \mathrm{~m}$ in favour of the PR group. Nevertheless, Marcellis et al. [18] reported similar findings to ours at T1. The differences in documented gain in walking distance may be explained by the slightly different settings, including length of the PR (12 vs. 3 weeks) and the demographics of the participants.

Physical reconditioning may occur due to disease-induced low daily exercise levels [6]. The physical training 
provided during PR seems to be the main reason for the improvements in the $6 \mathrm{MWD}$, especially since marginally reduced lung function values were only slightly improved at $\mathrm{T} 1$ and thus cannot be responsible for these results. The importance of muscular weakness in sarcoidosis patients has been frequently described in the literature $[6,8,15-17,40]$. Our results document a significant improvement in functional exercise capacity immediately following the 3-week PR programme, especially in the previously very impaired patients. These results are supported by a Cochrane analysis issued later [10]. Moreover, our results suggest that a 3-week inpatient PR programme shows benefits comparable to a 12- or 13week outpatient training programme regarding functional exercise capacity. This benefit of PR is particularly important, as several studies $[8,15,21]$ have shown that exercise intolerance is linked to reduced QoL, dyspnoea, and fatigue, thus frequently creating a persistent vicious circle, as has also been described for other pulmonary diseases $[5,6]$. PR can potentially break this circle by increasing exercise capacity and thus enhance physical activity levels in daily life [41]. Moreover, the greatest benefit of PR was seen in the severely exercise-intolerant patients.

The influences of obesity, steroid therapy, and medication changes on the PR results were small as no associations between the primary outcomes and these variables were found when using multivariate regression. Some of the comorbid disorders, such as depression, showed an association with QoL, an observation largely confirmed by other surveys on this topic.

No validated tool had been developed to measure sarcoidosis-specific HrQoL when this study was performed. Changes in HrQoL measured by the SGRQ in sarcoidosis PR patients are difficult to interpret as previous study designs were heterogeneous regarding setting, study population, and the tools used [22]. HrQoL in our study sample was clearly reduced on average, particularly in the SGRQ "activity" section, followed by the "symptoms" section. These findings are similar to those of Cox et al. [42], who reported an even greater reduction in HrQoL. In contrast to our study, Gvozdenovic et al. [43] and Antoniou et al. [44] described HrQoL in a hospital setting. Moreover, they assessed patients who were less severely affected by sarcoidosis than those in our study, thus the results cannot be compared directly. Marcellis et al. [18] used the WHOQOL-BREF to measure HrQoL and reported only significant improvements in psychological health, without any further differentiation. In 2007, Baughman et al. [21] found that the SGRQ activity com- ponent is an independent predictor of 6MWD. This corresponds to our findings regarding the activity component. The effect size of SRM $=0.62$ (total score) documented in ProKaSaRe is relatively comparable to that seen after rehabilitation in another group of patients with interstitial lung disease, which included sarcoidosis [3]. Nevertheless, the heterogeneity of sarcoidosis due to variations in other affected organs, functional impairment, and severity makes it difficult to identify one factor that is solely responsible for HrQoL improvement, even using the more recently suggested tools for QoL evaluation. The ProKaSaRe data, similarly to other recent findings [45], support the concept of PR with a multidimensional approach, consisting of other components besides exercise training, e.g., psychosocial support, patient education, improved understanding of the disease, its prognosis and therapeutic options, relaxation therapies, and breathing retraining [4]. Mutual influences and interlinked physiological training results, such as reduced dyspnoea (MRC), improved physical fitness (6MWD), or reduced fatigue contribute to the improvements in HrQoL after PR. Moreover, 6MWD and SGRQ scores were recently shown to have stronger associations with steps per day, while exercise capacity and QoL measurements were robust predictors of physical activity in patients with sarcoidosis [41]

At T1, small but significant changes were seen in VC and $\mathrm{FEV}_{1}$ while mean values of blood gases and $\mathrm{D}_{\mathrm{LCO}}$ were normal at T0 and T1. The average PI max showed the greatest improvement during PR. Kabitz et al. [38] and other authors suggest that inspiratory muscle weakness and impaired exercise capacity is more important when predicting dyspnoea and reductions in 6MWD than lung function and $\mathrm{D}_{\mathrm{LCO}}$. There is, however, no evidence in our data as to whether PR or inspiratory muscle training (IMT) improves PI max. Furthermore, the increase of PI max in our study sample was lower than in the only other available RCT concerning the benefit of IMT in sarcoidosis [46]. In our study, however, only a minority of the patients underwent IMT for 3 weeks, in contrast to the RCT of Karadalli et al. [46] where all patients in the treatment group were trained for a total of 30 $\mathrm{min} /$ day, 7 days/week, for 6 weeks. We found no association between the gains in PI max and benefits to other clinically more relevant outcomes such as exercise capacity, dyspnoea or QoL. The clinical relevance of the PI max increase and the influence of IMT in our study therefore remains unclear.

Although the MRC dyspnoea scale is not highly sensitive to changes, a small but significant improvement at T1 
was documented, which corresponds to the findings of Marcellis et al. [18]. This is presumed to be most likely due to the exercise training during $\mathrm{PR}$, although many other components of PR have the potential to influence patients' perception (and therefore evaluation) of dyspnoea, such as respiratory physiotherapy or solicited psychological and behaviour change. Some of the study participants may also have displayed a disproportionate dyspnoea related to dysfunctional breathing disorders. Unfortunately, no additional data are available to support any further hypothesis.

Fatigue is a frequent pathological symptom with an unclear aetiology [47]. It affects approximately $70 \%$ of patients with sarcoidosis in Germany, particularly women [48]. It is associated with reduced HrQoL [49], cognitive deficits, depression [50], and reduced physical fitness [14]. The FAS score in our study correlates with SGRQ, $6 \mathrm{MWD}$, and MRC dyspnoea scores. As no specific treatment is known for this often very debilitating symptom, the documented positive effect of PR in ProKaSaRe is particularly important for both clinicians and patients. Two previous studies found improved FAS scores [18]. The results of one study correspond almost exactly with our findings [17], supporting a causal relationship between physical training and improvement of fatigue as has already been demonstrated for other clinical conditions such as multiple sclerosis or cancer [47]. Nevertheless, a very recent investigation calculated a weaker association of objectively measured physical activity with fatigue than expected [41], concluding that in sarcoidosis, "fatigue might not preclude affected patients from being physically active, although this symptom is subjectively perceived as highly disabling" and positively correlated to physical activity [51].

Psychological status as indicated by the scores of the HADS was, on average, in the upper range of normal for anxiety and the borderline range for depression. In fact, clearly pathological scores were determined for $16.2 \%$ of ProKaSaRe patients regarding anxiety and $15.9 \%$ regarding depression, while borderline scores were determined for $30.4 \%$ of patients regarding anxiety and $28.4 \%$ regarding depression. This indicates that clinically relevant psychological disorders are not unusual for sarcoidosis PR patients. PR professionals should be aware of these concomitant symptoms or disorders and offer specially tailored treatment options. The high frequency of mental health complaints may be partly explained by the oral steroids taken by $48 \%$ of our patients and, furthermore, by frustration regarding physical limitations and uncertainty about the disease.

Sarcoidosis and Pulmonary Rehabilitation

\section{Strengths and Limitation of the Study}

This multicentre cohort study is not a randomised, assessor-blinded, controlled trial. Rehabilitation is a statutory right under German law and thus it is almost impossible to conduct RCTs. Instead, the ProKaSaRe study was designed to compile real-life data reflecting the current situation of rehabilitation for sarcoidosis. Exercise capacity should ideally be assessed using spiro-ergometry, which was not available at all centres. Additionally, skeletal muscle weakness, which has been demonstrated to be related to exercise intolerance [15] was not evaluated in the study, although this might have facilitated the discussion of changes in dyspnoea. Despite the high inclusion and questionnaire return rates there may be variations in treatment and some subgroups of sarcoidosis patients may be underrepresented in our sample. It is possible that patients selected and referred for PR by the authorities had a greater rehabilitation potential than those not selected. To remove this bias, we plan a follow-up randomised study using a waiting-list design in order to create an appropriate control group by assigning subjects at random to the rehabilitation or the waiting list [53]. In order to minimise known biases such as seasonal influences on the results we recruited continuously over the period of 1 year. We also performed statistical control of confounding variables to exclude assumed influences on the results such as weight loss during PR.

In Germany in the year 2011 a total of 929 patients with sarcoidosis were admitted to rehabilitation. Almost a third of all these patients were included in the multicentre ProKaSaRe study. The results can therefore be regarded as representative for PR in Germany. Nevertheless, the causal effects of PR are not certain, although the sample of 296 patients is, to our knowledge, the largest assessed for this purpose worldwide and our findings are strengthened by a 1-year follow-up.

\section{Summary}

ProKaSaRe describes the sarcoidosis patients referred for multimodal inpatient PR in Germany. It shows that they are younger and more often employed than PR patients in general, and that they have relevant limitations in HrQoL and exercise capacity that cannot be adequately assessed by lung function parameters alone. Additionally, ProKaSaRe provides initial evidence that PR leads to significant and clinically relevant improvements that can be measured directly after the rehabilitation. Our results support the recommendations of other studies and high- 
light the importance of individualised multimodal PR concepts $[4,7,52]$, while suggesting that training and rehabilitation are important and effective complementary therapies for the management of all degrees of burden of sarcoidosis. The extent of the positive effects of PR should be investigated further.

\section{Acknowledgements}

The authors would like to thank the retired directors of two of the participating centres, Dr. U. Tönnesmann and Dr. R.H. Heitmann, for their support and collaboration in the ProKaSaRe project, the participating patients for their willingness to contribute, the study nurses for their valuable assistance, and P. Kay Fedorov and R. Stark for their proof reading, all of which was really appreciated. The study was supported by an unrestricted grant from the Deutsche Lungenstiftung e.V. and the Sarkoidose Stiftung.

\section{References}

1 Statement on sarcoidosis. Joint Statement of the American Thoracic Society (ATS), the European Respiratory Society (ERS), and the World Association of Sarcoidosis and Other Granulomatous Disorders (WASOG) adopted by the ATS Board of Directors and by the ERS Executive Committee, February 1999. Am J Respir Crit Care Med 1999:160:736755 .

-2 Bosse-Henk A, Koch R, Wirtz H, Hinz A: Fatigue and excessive daytime sleepiness in sarcoidosis: prevalence, predictors, and relationships between the two symptoms. Respiration 2017;94:186-197.

3 Spruit MA, Singh SJ, Garvey C, ZuWallack R, Nici L, Rochester C, Hill K, Holland AE, Lareau SC, Man WD, et al: An official American Thoracic Society/European Respiratory Society statement: key concepts and advances in pulmonary rehabilitation. Am J Respir Crit Care Med 2013;188:e13-e64.

4 Osadnik CR, Rodrigues FMM, Camillo CA, Loeckx M, Janssens W, Dooms C, Troosters T: Grundlagen der Rehabilitation und Reaktivierung. Karger Kompass Pneumol 2016;4: 8-17.

5 Langer D: Rehabilitation in patients before and after lung transplantation. Respiration 2015;89:353-362.

6 Burtin C, Hebestreit H: Rehabilitation in patients with chronic respiratory disease other than chronic obstructive pulmonary disease: exercise and physical activity interventions in cystic fibrosis and non-cystic fibrosis bronchiectasis. Respiration 2015;9:181-189.

-7 Dreher M, Ekkernkamp E, Schmoor C, Schoenheit-Kenn U, Winterkamp S, Kenn K: Pulmonary rehabilitation and noninvasive ventilation in patients with hypercapnic interstitial lung disease. Respiration 2015;89: 208-213.

8 Holland AE, Dowman LM, Hill CJ: Principles of rehabilitation and reactivation: interstitial lung disease, sarcoidosis and rheumatoid disease with respiratory involvement. Respiration 2015;89:89-99.
9 Holland AE, Hill CJ, Conron M, Munro P, McDonald CF: Short-term improvement in exercise capacity and symptoms following exercise training in interstitial lung disease. Thorax 2008;63:549-554.

10 Dowman L, Hill CJ, Holland AE: Pulmonary rehabilitation for interstitial lung disease. Cochrane Database Syst Rev 2014; 10: CD006322.

-11 Ferreira A, Garvey C, Connors GL, Hilling L, Rigler J, Farrell S, Cayou C, Shariat C, Collard HR: Pulmonary rehabilitation in interstitial lung disease: benefits and predictors of response. Chest 2009;135:442-447.

12 Huppmann P, Sczepanski B, Boensch M, Winterkamp S, Schonheit-Kenn U, Neurohr C, Behr J, Kenn K: Effects of inpatient pulmonary rehabilitation in patients with interstitial lung disease. Eur Respir J 2013;42:444-453.

13 Marcellis RG, Lenssen AF, de Vries J, Drent M: Reduced muscle strength, exercise intolerance and disabling symptoms in sarcoidosis. Curr Opin Pulm Med 2013;19:524-530.

14 Marcellis RG, Lenssen AF, Elfferich MD, De Vries J, Kassim S, Foerster K, Drent M: Exercise capacity, muscle strength and fatigue in sarcoidosis. Eur Respir J 2011;38:628-634.

15 Spruit MA, Thomeer MJ, Gosselink R, Troosters T, Kasran A, Debrock AJ, Demedts MG, Decramer M: Skeletal muscle weakness in patients with sarcoidosis and its relationship with exercise intolerance and reduced health status. Thorax 2005;60:32-38.

16 Drent M, Marcellis R, Lenssen A, De Vries J: Association between physical functions and quality of life in sarcoidosis. Sarcoidosis Vasc Diffuse Lung Dis 2014;31:117-128.

17 Strookappe B, Swigris J, De Vries J, Elfferich M, Knevel T, Drent M: Benefits of physical training in sarcoidosis. Lung 2015;193:701-708.

18 Marcellis R, Van der Veeke M, Mesters I, Drent M, De Bie R, De Vries G, Lenssen A: Does physical training reduce fatigue in sarcoidosis? Sarcoidosis Vasc Diffuse Lung Dis 2015;32:53-62.

19 Strookappe B, Saketkoo LA, Elfferich M, Holland A, De Vries J, Knevel T, Drent M: Physical activity and training in sarcoidosis: review and experience-based recommendations. Expert Rev Respir Med 2016;10:1057-1068.
20 Jones PW, Quirk FH, Baveystock CM, Littlejohns P: A self-complete measure of health status for chronic airflow limitation. The St. George's Respiratory Questionnaire. Am Rev Respir Dis 1992;145:1321-1327.

21 Baughman RP, Sparkman BK, Lower EE: Sixminute walk test and health status assessment in sarcoidosis. Chest 2007;132:207-213.

22 De Vries J, Drent M: Quality of life and health status in sarcoidosis: a review of the literature. Clin Chest Med 2008;29:525-532.

23 Jones PW: Interpreting thresholds for a clinically significant change in health status in asthma and COPD. Eur Respir J 2002;19:398404.

24 Holland AE, Spruit MA, Troosters T, Puhan MA, Pepin V, Saey D, McCormack MC, Carlin BW, Sciurba FC, Pitta F, et al: An official European Respiratory Society/American Thoracic Society technical standard: field walking tests in chronic respiratory disease. Eur Respir J 2014;44:1428-1446.

25 Alhamad EH: The six-minute walk test in patients with pulmonary sarcoidosis. Ann Thorac Med 2009;4:60-64.

26 Bullinger M: German translation and psychometric testing of the SF-36 Health Survey: preliminary results from the IQOLA Project. International Quality of Life Assessment. Soc Sci Med 1995;41:1359-1366.

27 De Vries J, Michielsen H, Van Heck GL, Drent M: Measuring fatigue in sarcoidosis: the Fatigue Assessment Scale (FAS). Br J Health Psychol 2004;9:279-291.

28 Herrmann C, Buss U, Snaith RP: HADS-D Hospital Anxiety and Depression Scale Deutsche Version: Ein Fragebogen zur Erfassung von Angst und Depressivität in der somatischen Medizin. Bern, Huber, 1995.

29 Criee CP, Berdel D, Heise D, Kardos P, Kohler D, Leupold W, Magnussen H, Marek W, Merget R, Mitfessel H, et al: Recommendations on spirometry by Deutsche Atemwegsliga (in German). Pneumologie 2006;60:576-584.

30 Criee CP: Whole-body plethysmography (in German). Med Klin (Munich) 2010;105:652660 
- 31 Bestall JC, Paul EA, Garrod R, Garnham R, Jones PW, Wedzicha JA: Usefulness of the Medical Research Council (MRC) dyspnoea scale as a measure of disability in patients with chronic obstructive pulmonary disease. Tho$\operatorname{rax}$ 1999;54:581-586.

32 Lieberman J: Elevation of serum angiotensinconverting-enzyme (ACE) level in sarcoidosis. Am J Med 1975;59:365-372.

33 Lingner H, Grosshennig A, Flunkert K, BuhrSchinner H, Heitmann R, Tonnesmann U, van der Meyden J, Schultz K: ProKaSaRe Study Protocol: a prospective multicenter study of pulmonary rehabilitation of patients with sarcoidosis. JMIR Res Protoc 2015; 4:e134.

34 Scadding JG: Prognosis of intrathoracic sarcoidosis in England. A review of 136 cases after five years' observation. Br Med J 1961;2: 1165-1172.

35 de Kleijn WP, De Vries J, Wijnen PA, Drent M: Minimal (clinically) important differences for the Fatigue Assessment Scale in sarcoidosis. Respir Med 2011;105:1388-1395.

36 Enright PL, Sherrill DL: Reference equations for the six-minute walk in healthy adults. Am J Respir Crit Care Med 1998;158:1384-1387.

- 37 Bourbonnais JM, Malaisamy S, Dalal BD, Samarakoon PC, Parikh SR, Samavati L Distance saturation product predicts healthrelated quality of life among sarcoidosis patients. Health Qual Life Outcomes 2012, 10:67.

38 Kabitz HJ, Lang F, Walterspacher S, Sorichter S, Muller-Quernheim J, Windisch W: Impact of impaired inspiratory muscle strength on dyspnea and walking capacity in sarcoidosis. Chest 2006;130:1496-1502.
39 Alhamad EH, Shaik SA, Idrees MM, Alanezi MO, Isnani AC: Outcome measures of the 6 minute walk test: relationships with physiologic and computed tomography findings in patients with sarcoidosis. BMC Pulm Med 2010;10:42.

40 Marcellis RG, Lenssen AF, Kleynen S, De Vries J, Drent M: Exercise capacity, muscle strength, and fatigue in sarcoidosis: a followup study. Lung 2013;191:247-256.

41 Bahmer T, Kirsten AM, Waschki B, Rabe KF, Magnussen H, Kirsten D, Gramm M, Hummler S, Brunnemer E, Kreuter M, Watz H: Clinical correlates of reduced physical activity in idiopathic pulmonary fibrosis. Respiration 2016;91:497-502.

42 Cox CE, Donohue JF, Brown CD, Kataria YP, Judson MA: Health-related quality of life of persons with sarcoidosis. Chest 2004;125: 997-1004.

43 Gvozdenovic BS, Mihailovic-Vucinic V, IlicDudvarski A, Zugic V, Judson MA: Differences in symptom severity and health status impairment between patients with pulmonary and pulmonary plus extrapulmonary sarcoidosis. Respir Med 2008;102:1636-1642.

44 Antoniou KM, Tzanakis N, Tzouvelekis A, Samiou M, Symvoulakis EK, Siafakas NM, Bouros D: Quality of life in patients with active sarcoidosis in Greece. Eur J Intern Med 2006; 17:421-426.

45 McCarthy B, Casey D, Devane D, Murphy K, Murphy E, Lacasse Y: Pulmonary rehabilitation for chronic obstructive pulmonary disease. Cochrane Database Syst Rev 2015; 2:CD003793.
46 Karadalli MN, Bosnak-Guclu M, Camcioglu B, Kokturk N, Turktas H: Effects of inspiratory muscle training in subjects with sarcoidosis: a randomized controlled clinical trial. Respir Care 2016;61:483-494.

47 Drent M, Lower EE, De Vries J: Sarcoidosisassociated fatigue. Eur Respir J 2012;40:255263

48 Hinz A, Fleischer M, Brahler E, Wirtz H, Bosse-Henck A: Fatigue in patients with sarcoidosis, compared with the general population. Gen Hosp Psychiatry 2011;33:462-468.

49 de Kleijn WP, De Vries J, Lower EE, Elfferich MD, Baughman RP, Drent M: Fatigue in sarcoidosis: a systematic review. Curr Opin Pulm Med 2009;15:499-506.

50 Elfferich MD, Nelemans PJ, Ponds RW, De Vries J, Wijnen PA, Drent M: Everyday cognitive failure in sarcoidosis: the prevalence and the effect of anti-TNF- $\alpha$ treatment. Respiration 2010;80:212-219.

51 Bahmer T, Kirsten AM, Waschki B, Rabe KF, Magnussen H, Kirsten D, Gramm M, Hummler S, Brunnemer E, Kreuter M, Watz H: Clinical correlates of reduced physical activity in idiopathic pulmonary fibrosis. Respiration 2016;91:497-502.

52 Osadnik CR, Rodrigues FMM, Camillo CA, Loeckx M, Janssens W, Dooms C, Troosters T: Principles of rehabilitation and reactivation. Respiration 2015;89:2-11.

53 Schultz K, Seidl H, Jelusic D, Wagner R, Wittmann M, Faller H, Nowak D, Schuler M: Effectiveness of pulmonary rehabilitation for patients with asthma: study protocol of a randomized controlled trial (EPRA). BMC Pulm Med 2017;17:49-59. 